RESEARCH NOTE

\section{The Tegument of Schistosoma mansoni: Genes, Antigens and the Host-Parasite Relationship}

\section{Edneide M Xavier, Norma Lucena- Silva, Roberto P Werkhauser, Gloria R Franco*, Ricardo AA Lira Santos, Andrew JG Simpson**, Frederico GC Abath ${ }^{+}$}

Departamento de Imunologia, Centro de Pesquisas Aggeu Magalhães-Fiocruz, Av. Moraes Rego s/n, Cidade Universitaria, 50670-420, Recife, PE, Brasil *Departamento de Bioquímica, ICB, Universidade Federal de Minas Gerais, Belo Horizonte, MG, Brasil **Instituto Ludwig de Pesquisas sobre o Câncer, Rua Prof. Antonio Prudente 109, 01509-010, São Paulo, SP, Brasil

Key words: Schistosoma mansoni - tegument - genes antigens

Cercaria, the infective larvae of Schistosoma mansoni are released from a molluscan host and must penetrate the skin of the human host within several hours in order to continue the life-cycle of the parasite. Thus, after release, the parasite progresses from a transient life in fresh water to a new parasitic existence initially in the skin and subsequently in the bloodstream of the host. The parasite undergoes adaptative changes to effect this transition, many of them at the level of the tegument (FGC Abath \& RP Werkhauser 1996 Parasite Immunol 18: 15-20). Although some of these alterations constitute mechanisms to avoid the immune defense, paradoxically, many antigens, targets of the immune response against the schistosome, are also associated with the tegument (SR Smithers et al. 1989 Parasite Immunol 11: 301318).

This work was funded by WHO, FACEPE and CNPq. Corresponding author. Fax: +55-81-453.1911. E-mail: fabath@gene.dbbm.fiocruz.br

Received 4 May 1998

Accepted 31 August 1998
The tegument of the adult worm consists of a cytoplasmic syncitium enclosed by a unique double outer tegumental membrane (DJ McLaren \& KJ Hockley 1977 Nature 269: 147-149). The tegumental outer membrane of $S$. mansoni, which consists of two closely apposed lipid bilayers, extends over the whole of the pitted surface of the tegument, including the spines.

The identification and characterization of proteins associated with the tegument is central to our understanding of the function of this structure, and also provides probes for defining the mechanisms of membrane formation. However, research in this area has mainly been stimulated by the search for molecules that may act as targets of protective immune responses or that could be relevant for diagnosis. In this context, it has been shown that isolated tegumental membranes are capable of stimulating protective immunity in mice (Smithers et al. 1989 loc. cit., 1990 Parasitol Res 76: 454456). The principal membrane associated antigens of the tegument of adult $S$. mansoni cross react with the younger schistosomular forms of the parasite which are the targets of protective immunity (AJG Simpson 1990 Parasitol Today 6: 40-45).

Several genes for major antigens recognized by antibodies from animals protectively immunized with tegumental membranes (Smithers et al. 1990 loc. cit.) have been cloned: Sm25 (P Omer-Ali et al. 1991 Mol Biochem Parasitol 45: 215-222), Sm22 (SA Jeffs et al. 1991 Mol Biochem Parasitol 46: 159-168), Sm15 (FGC Abath et al. $1993 \mathrm{Mol}$ Biochem Parasitol 60: 81-92), Sm13 (manuscript in preparation) and Sm8. Although they are potentially important for protective immunity in the mouse model, direct vaccination experiments with the recombinant proteins are required and are currently being carried out.

The cDNAs for Sm15 (A70 cDNA), Sm13 (A157 cDNA) and Sm8 (A142 cDNA) have been isolated by screening a (lt11 adult $S$. mansoni cDNA library with rabbit anti-adult worm tegumental membrane antisera $(\mathrm{RaM})$. The identification of the antigens encoded by the cDNAs was undertaken by selecting antibodies specific for the expressed b-galactosidase fusion proteins from RaM. Genomic Southern blot analysis is consistent with each of the corresponding genes being present in a single copy. There is evidence suggesting that the gene for Sm15 encodes a precursor differentially processed during parasite maturation (FGC Abath et al. 1994 Parasitol Res 80: 64-69). The complete gene for $\mathrm{Sm} 13$ was obtained from genomic and cDNA fragments. For $\mathrm{Sm} 8$, only a partial cDNA is currently available (the 5 'end is missing). Recently, we have subcloned and expressed the genes for Sm13 and Sm8 in pMal-cri 
(New England Biolabs). Antibodies have been raised against MBP-rSm13 fusions and vaccinations experiments are under way.

The developmental expression of these tegumental antigens has been examined by Northern and Western blotting. The gene for the precursor of Sm15 and the gene for $\mathrm{Sm} 8$ have similar patterns of mRNA expression, being expressed in miracidia and sporocysts, but predominantly in adult worms. However, the gene for $\mathrm{Sm} 13$ is expressed only in adult worms. Western blot analysis of different life cycle stages of the parasite, using antibodies affinity purified from anti-adult worm tegumental membranes suggest that more than one antigenic product (23 and $15 \mathrm{kDa}$ ) are expressed in adult worms from the gene for the Sm15 precursor, and that Sm15 is not present in either schistosomula or miracidia (Abath et al. 1994 loc. cit.). The developmental expression of $\mathrm{Sm} 13$ has been undertaken using antibodies against maltose binding protein (MBP)-rSm13 fusions. A detailed examination of the kinetics of expression of Sm13 showed the same pattern as Sm15 (manuscript in preparation). The kinetics of expression of Sm8 has not been studied in detail. However, like the two other antigens it is detected in adult worms but not in 3-h schistosomulum. Thus the genes for these proteins are regulated during development and are coexpressed in the tegument of adult worms.

The hydrophilicity plot of Sm15 does not suggest the presence of any hydrophobic membranespanning segments. However, Sm15 is present in preparations enriched for tegumental membranes and separates to the detergent phase of Triton X114 , a behaviour common to integral membrane proteins (Abath et al. 1994 loc. cit.). Both Sm13 and $\mathrm{Sm} 8$ behave as integral membranes in TritonX 114 partition experiments. In contrast to Sm15 the derived amino acid sequence of these genes support these experimental findings, as they have putative transmembrane domains.

It is unclear how proteins that are predicted to be cytosolic, such as Sm15 (Abath et al. 1994 loc. cit.), GST (glutathione S-transferase, JM Balloul et al. 1987 Nature 326: 149-153), TPI (triose-phosphate isomerase, DA Harn et al. 1992 J Immunol 148: 562-567), GAPDH (glyceraldehyde-3-phosphate dehydrogenase, V Goudot-Crozel et al. 1989 $J$ Exp Med 170: 2065-2080) and PGK (phosphoglycerate kinase, KW Lee et al. $1995 \mathrm{Mol}$ Biochem Parasitol 71: 221-231), could be attached to the schistosome surface, since none has a conventional transmembrane domain and there is no evidence of a lipid anchor. One possiblity is that the parasite is damaged during the course of an infection and soluble proteins are adsorbed to the surface perhaps as part of its strategy to acquire host antigens that act as an antigenic disguise [AJG Simpson 1992 Mem Inst Oswaldo Cruz 87 (suppl. IV): 1117]. On the other hand the adsorption of soluble proteins derived from the parasite to the surface seems to be relevant for stimulation of a strong protective immunity (as is the case of the vaccine candidates GST, TPI, GAPDH and PGK). Interestingly, there is a striking accumulation of actin in areas of tegument recovering from damage (Y Matsumoto et al. 1989 Nature 333: 76-78). Thus, possibly, actin or other tegumental proteins are capable of interacting with some soluble proteins allowing attachment to the tegumental surface. We are currently investigating possible mechanisms of protein adsorbtion to the tegumental surface and the interaction of protein species within the tegumental membrane. We believe that this line of investigation is central to understanding how the schistosome survives within the intravascular habitat.

Acknowledgments: to Mr Rafael Guimarães and Mrs Rosineide Lira for technical assistance. 